\title{
Variations of Total Phenolic Content in Honey Samples Caused by Different Calibration Lines
}

\author{
(1) Drago Bešlo, ${ }^{1, *}$ Klara Bešlo, ${ }^{2}$ (b) Dejan Agić, ${ }^{1}$ (1) Dražen Vikić-Topić, ${ }^{3,4}$ () Bono Lučić4,\#
}

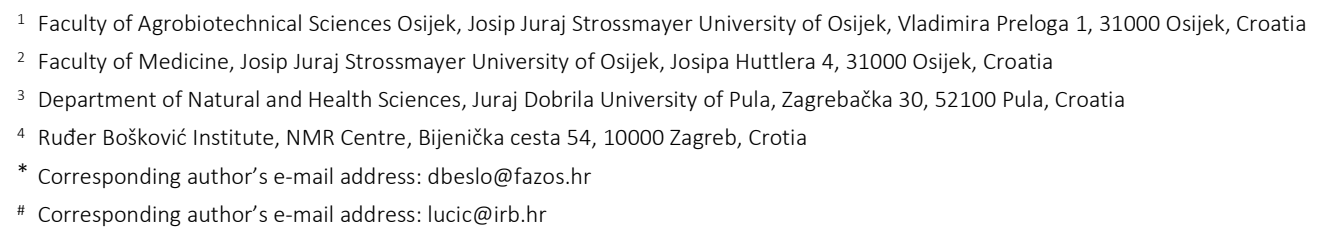

RECEIVED: May 30, 2021 * REVISED: September 10, 2021 * ACCEPTED: September 11, 2021

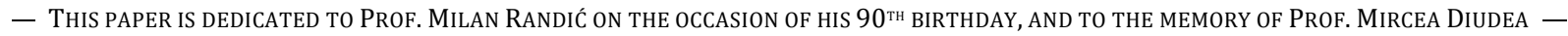

\begin{abstract}
In the review of total phenolic contents (TPCS) of acacia, lime, and chestnut honey samples from several literature sources, large differences were noticed, which cannot be attributed only to seasonal or geographical variations. The dependence of TPC on the process of construction of the calibration line is illustrated in the measurement of acacia, lime, and chestnut honey types from Croatia and neighbouring countries (Serbia, Italy, and Hungary). TPCs are determined for 39 uni-floral honey samples by four calibration lines and four TPC values are obtained for each honey sample. Obtained results are compared mutually, as well as with the literature results for honey samples of the same type. For each honey type, the average of all determined TPCs determined in this study is in the middle of literature values. The average TPC values for chestnut honey samples were found to be 1.5 and 3 times higher than those for lime and acacia, respectively. The effects of two factors regularly considered in the determination of calibration lines are analyzed: (1) the concentration range of the standard chemical and (2) whether the calibration line is drawn through the origin, or not. The final results strongly depend on these two factors that should be considered in future TPC estimations.
\end{abstract}

Keywords: honey; total phenolic content; calibration line; protocol standardization; spectrophotometric method.

\section{INTRODUCTION}

$\mathbf{H}$ ONEY has been used since the earliest times as natural food produced by honeybees (Apis mellifera $L$ ) from the nectar of blossoms or exudates of trees and plants. It is recognized that honey has various biological properties ${ }^{[1]}$ contributing beneficially to the health of human organisms. ${ }^{[2]}$ The contents of minerals and natural chemicals in honey depend on various factors such as the season conditions, floral type, geographical origin, or storage, and processing conditions. ${ }^{[3-5]}$ The focus of most analyses of honey content is mainly concentrated on the content of polyphenols, which is measured as the total phenolic content. ${ }^{[6,7]}$ Polyphenols are natural phytochemical products of secondary plant metabolism having diverse biological effects, such as antiinflammatory, anti-carcinogenic, and anti-atherosclerotic effects, as a result of their antioxidant activity ${ }^{[1]}$ or ability to modify cellular signalling pathways. ${ }^{[2]}$ Among polyphenols present in honey, flavonoids and phenolic acids are identified as the most important groups of components showing antioxidant activity. ${ }^{[6,7]}$ Higher content of polyphenols in honey and other natural food products raises their nutritional quality and, consequently, could increase the honey market price. The composition of phenolic compounds in honey is influenced by floral sources of honey, seasonal and climate factors, and processing and storing conditions. The content of nectar of individual plant species in honey varies depending on the type of vegetation, the flowering period of plant species, as well as the time when the beekeeper produced the honey. Absolute uni-floral honey does not exist and, therefore, the chemical composition and organoleptic properties between honey samples can vary significantly. 
There are different methods used for the determination of the content of total polyphenols, and the identification of the presence of a polyphenol (flavonoid or phenolic acid) in honey samples. ${ }^{[8]}$ The aim of this study is to determine the total phenol content (TPC) in three groups of honey samples from South-Eastern European countries (Croatia, Serbia, Hungary, and Italy) using the spectrophotometric method based on the Folin-Ciocalteu reagent. ${ }^{[9]}$ TPC of honey has been predominantly determined by spectrophotometric methods that are simple, fast, and cost-effective. These methods are not selective, likely providing overestimated values, especially when using crude honey. ${ }^{[10,11]}$ The most common method for TPC estimation is based on a modification of the Folin-Ciocalteu procedure, ${ }^{[12-15]}$ which is very unspecific. The first work that applied the procedure of determining TPC in biological samples such as honey samples is the work of Meda et al..16] Later, Beretta et al. recognized the need for the standardization of measurements of antioxidant properties of honey by a combination of spectrophotometric/ fluorimetric assays and chemometrics analysis, ${ }^{[17]}$ and this modification was also used by Bertoncelj et al. ${ }^{[6]}$ These two mentioned studies $^{[16,17]}$ are frequently used and cited by researchers, and it is also evident that they have been predominantly used for TPC measurements of honey samples. The reasons for large variations in TPC values can be different, such as seasonal variations, regional origin, honey storage methods, the correctness of analysis, etc. Analysing the results of measurements of TPC values of honey samples, we noticed that the procedures for constructing calibration lines are insufficiently well described in works measuring TPC of honey samples - where usually scanty ${ }^{[6,16,17]}$ or even no data are given. ${ }^{[18]}$ On the other hand, it is known from statistical analysis that TPC values estimated from the calibration line can vary significantly depending on the procedure (protocol) used in its construction.

However, by literature overview, we noticed that the TPC values of honey samples vary significantly, even though they come from the same country or region. The reasons for high variations in TPC values can be different, as mentioned above. By the analysis and literature overview of measurements of TPC values of different honey samples we found that:

(1) procedures used for construction of calibration line have not been sufficiently well described in studies measuring TPC honey samples where scanty data are usually given, ${ }^{[6,16,17]}$ and

(2) TPC values can vary considerably depending on how the calibration curve/line is constructed.

In this paper, we present the results of analysis of variability of readings of TPC value that can occur in two cases: (1) when the calibration line is determined so that it passes through the origin, and (2) when the line is constructed in such a way that it does not have to pass through the origin (i.e. has a constant term). The dependence of variation of TPC values on these two mentioned factors was investigated in measurements on uni-floral samples of acacia, lime, and chestnut honey. Doing so, we minimize the influence of floral origin variation that can be much larger in cases of multi-floral honey samples. The results obtained in this analysis on unifloral honey samples indicate the importance of this issue, and the need to provide detailed data and precise information on the process of determination of TPC values (in general - for all samples). Moreover, all important details related to the construction of the calibration line used in the determination of TPC values and its complete equation should be provided.

\section{MATERIALS AND METHODS}

\section{Honey Samples}

Three groups of samples of Apis mellifera honey obtained directly from beekeepers from different regions were acquired in the South Europe (Northern Italy, Hungary, Croatia, and Serbia) region in 2017. There were 19 samples of the acacia type, 12 chestnuts, and eight lime. Before and during the analyses, all samples were stored in glass containers in the dark at room temperature. ${ }^{[15]}$ Pollen and organoleptic analysis of samples were carried out as a part of the competition organized by the Association of Beekeepers of Slavonia and Baranja "Radilica" from Osijek as a part of $9^{\text {th }}$ international and $11^{\text {th }}$ regional competition in Osijek in August 2017 (https://www.hgk.hr/countychamber-osijek/ days-of-honey-in-croatia-announcement). All honey samples were received from July 25 to August 5, 2017 , and performed chemical analyses in the period from September 1-20, 2017.

\section{Determination of Total Phenolic Content}

Samples were prepared according to the slightly modified method proposed by Meda et al. ${ }^{[16]}$ Each honey sample $(15 \mathrm{~g})$ was mixed with $15 \mathrm{ml}$ of ultrapure water, homogenized by vortex mixing, transferred to a $50 \mathrm{ml}$ volumetric flask, and filled with ultrapure water. This solution $(0,1 \mathrm{ml})$ was then mixed with $1 \mathrm{ml}$ of $10 \%$ Folin-Ciocalteu reagent (Kemika, Zagreb, Croatia) for $5 \mathrm{~min}$ and $1 \mathrm{ml}$ of $75 \mathrm{~g} / \mathrm{L}$ sodium carbonated $\left(\mathrm{Na}_{2} \mathrm{CO}_{3}\right)$ (Kemika, Zagreb, Croatia) was then added. The reaction was carried out in a dark place at room temperature. After incubation at room temperature for $2 \mathrm{~h}$, the absorbance of the reaction mixture was measured at $750 \mathrm{~nm}$ against a methanol blank. Three parallel absorbance-concentration determinations for each sample were averaged and taken as the final result. TPC values of honey samples were expressed in $\mathrm{mg}$ of 
gallic acid (GA) per kg of honey (GAE, Gallic Acid Equivalent) using the $\mathrm{GA}$ calibration line in the range $(0-400$ $\mathrm{mg} / \mathrm{L}$ or $0-200 \mathrm{mg} / \mathrm{L}$ ).

\section{Statistical Analysis}

Data and correlation analysis and line-fitting have been done by the Microsoft Excel program. All figures, as well as the calibration line determination and verification, were created by the Origin program (OriginLab Corp., Northampton, MA, USA). In the calibration analysis, only linear function (a first-degree polynomial, i.e. a straight line) was considered, since the relationship between gallic acid (GA) concentrations and absorbances is linear in the interval $0-250 \mathrm{mg} / L{ }^{[17]}$ but also larger intervals of linearity $0-500 \mathrm{mg} / \mathrm{L}^{[18]}$ and $0-1000 \mathrm{mg} / \mathrm{L}$ have reported. ${ }^{[19]}$

The GA concentration interval used for the determination of the calibration line with the number of replications has been commonly reported in the literature. ${ }^{[5,6,15-21]}$ Only sporadically, the correlation coefficient $(r)$ or its square has been given in the literature as a statistical measure of the quality of fitting between the absorbances and the corresponding GA concentrations. ${ }^{[17,19]}$ The quality of the fit of data by calibration lines is expressed by the correlation coefficient $(r)$ and the standard error of estimate (S), which is the root mean squared error between the absorbances calculated by the calibration line and the corresponding experimental absorbance values.

\section{RESULTS AND DISCUSSION}

\section{Construction of TPC Calibration Lines}

TPC values were measured for 39 honey samples by the described protocol that is a slight modification of the one introduced by Meda et al..[16] In order to obtain a stronger absorbance signal for the least active samples (acacia), the TPC determination was performed from $15 \mathrm{~g}$ of honey samples instead from $5 \mathrm{~g}$ as in the original method..[16] For each sample, four TPC values are estimated each time using one of four GAE calibration lines. Two calibration equations (lines) were determined, giving the relationship between the concentration ( $x$-axis) and the absorbance ( $y$-axis) of $\mathrm{GA}$. The first applies to the concentration interval 0.02-0.2 $\mathrm{g} / \mathrm{L}(20-200 \mathrm{mg} / \mathrm{L})$ and the second to the interval 0.04$0.4 \mathrm{~g} / \mathrm{L}$ of $\mathrm{GA}$. Furthermore, for each interval the calibration line was determined in two ways:

(1) the equation of the straight line is determined without limitation and may have an intercept $(l)$ on the $y$-axis of the general form $y=k x+l$, and

(2) provided that the straight line have to pass through the origin (i.e. that the line has no intercept on the $y$-axis: $y=k x$ ).
Thus, four calibration lines are determined, and each of which was applied to estimate the GA equivalent TPC value of the honey sample (expressed as $\mathrm{mg}$ of $\mathrm{GA} / \mathrm{kg}$ ). We obtained these two equations for the GA concentration interval $0.02-0.2 \mathrm{~g} / \mathrm{L}$ (Eqs. (1) and (2)).

$$
\begin{aligned}
& y=5.2903 x+0.036 \\
& r=0.9995, s=0.104 \\
& y=5.5474 x \\
& r=0.9995, \quad s=0.201
\end{aligned}
$$

The position of both calibration lines relative to the points defined by the experimentally measured absorbances and the concentrations of gallic acid (Table S1, Supplementary information) is shown in Figure 1. From the mutual position of these lines, one can see that the differences are the largest at both ends of the concentration interval being minimal in the middle.

It looks like both lines are equally good. Namely, both Eqs. (1) and (2) have very high correlation coefficients $(r>0.9995)$. The information on almost identical and very high correlation coefficients can be misleading, and this is the only parameter (or its square) given in the literature ${ }^{[17-}$ ${ }^{20]}$ with the calibration lines as an indicator of fitting quality. Although, we can see from Eqs. (1) and (2) that the differences of standard errors of estimate $S$ are much larger (0.104 for Eg. (1) vs 0.201 for Eg. (2)), indicating that the calibration line having $y$-intercept is the better one (i.e. more accurate comparing with experimental values).

Further, equations of two calibration lines for the estimation of the GA equivalent TPC value from the measured absorbance of the honey sample ( $\mathrm{mg} \mathrm{GA} / \mathrm{kg}$ of sample), established in the wider GA concentration interval $0.04-0.4 \mathrm{~g} / \mathrm{L}(4-400 \mathrm{mg} / \mathrm{L})$, are given by Eqs. (3) and (4):

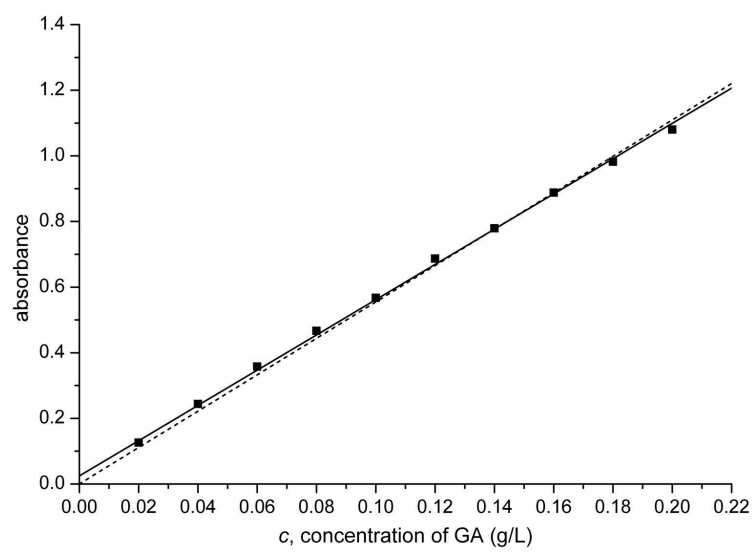

Figure 1. Gallic acid calibration lines in the concentration range $0.02-0.2 \mathrm{~g} / \mathrm{L}$. Solid straight line corresponds to Eq. (1), and the dotted line is for Eq. (2). 


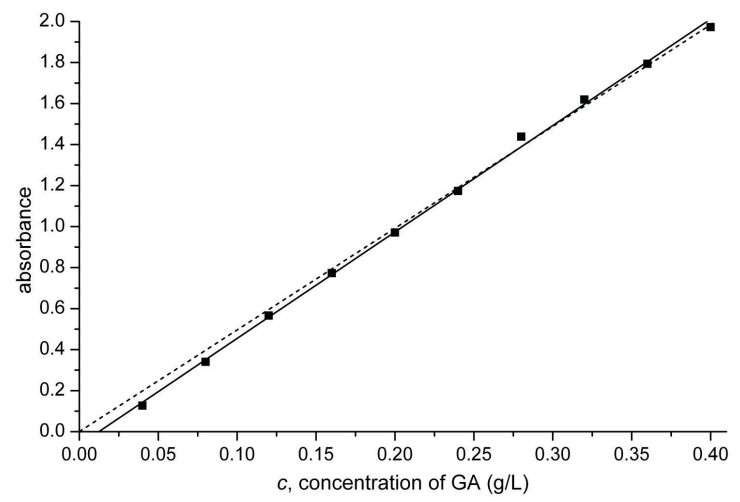

Figure 2. Gallic acid calibration lines in the concentration range $0.04-0.4 \mathrm{~g} / \mathrm{L}$. Solid straight line corresponds to Eq. (3), and the dotted line is for Eq. (4).

$$
\begin{aligned}
& y=5.1898 x-0.0642 \\
& r=0.9993, \quad S=0.025 \\
& y=4.9604 x \\
& r=0.9995, \quad s=0.039
\end{aligned}
$$

These calibration lines are given and their mutual position is shown in Figure 2. For each GA sample, three measurements of GA absorbances and their average value are given in Table S1 (Supplementary Information). Similar to the calibration lines shown in Figure 1, the correlation coefficients are very similar (i.e. almost identical), while there is a significant difference between the standard errors of the lines given by Eqs. (3) and (4).

It can be seen that the differences between these two calibration lines are larger at the lower range of concentrations. Also, the differences at lower concentrations are larger in Figure 2 than between calibration lines in Figure 1 that are constructed on a narrower GA concentration interval $0.02-0.2 \mathrm{~g} / \mathrm{L}$. This finding indicates that a larger difference will be observed between estimated TPC values using these two lines at lower concentrations. Because of that, the comparisons between TPC measurements made in different laboratories for the same honey samples will differ significantly when estimated TPC values are lower (i.e. when read at lower sample concentrations).

\section{Measurement and Determination of Total Phenolic Content}

The influence of concentration intervals and types of calibration (straight) lines on the estimated TPC values of 39 honey samples was analysed. For each honey sample, four TPC values are estimated using calibration lines given by Eqs. $(1-4)$. These TPC values, together with the corresponding absorbance values determined in triplicate, are given in Table S2 (Supplementary Information). The information on the honey floral origin, producer, and country are also listed for each sample in Table S2, as well as the range of TPC values for each honey floral type. Scattering of TPC values (mg GAE/ $\mathrm{kg}$ honey) for acacia, chestnut and lime honey samples determined using four calibration lines given by Eqs. $1-4$ are shown in Figures S1, S2 and S3, respectively (Supplementary Information). Mean TPC values and the corresponding standard deviations determined in this study are summarized in Table 1 and given (separately) for each type of honey.

Additionally, in the second part of Table 1 the corresponding TPC values from literature are given for the same honey types (acacia, chestnut, and lime) and for samples originated from Croatia and Italy that are included in our study. Furthermore, we add in Table 1 TPCs of samples originated from neighbouring countries (Slovenia, Romania, Czechia, and Turkey). In such a way the differences in TPC values due to the floral and regional variation of honey samples are minimized. Our measurements for three types of uni-floral honey samples are consistent and show no significant variation regarding the regional (country) origin of individual samples (see graphical illustration in Figures S1-S3). A possible important reason is that all measurements were carried out in the same laboratory, and the conditions for collecting and analysing samples were well defined and performed in a short period of time. Otherwise, comparisons between studies done on samples from the same country (or between countries) are regularly based on studies completed in different laboratories, which may apply different approaches in the construction of calibration lines leading to larger deviations.

Generally, significant variations of the corresponding TPC values read from four calibration lines were observed for each sample, and between mean values and standard deviations for samples of the same floral origin (Table 1 and Table S2). An even larger scattering of TPC values is evident between the literature data and the results of this study. More detailed inter-dependencies of determined TPC values and their comparison with TPC values from the literature are described below.

The total phenolic content of honey has been largely determined by spectrophotometric methods that are simple, fast, and cost-effective. This method is not suitable for the analysis of crude honey because, in such a case, overestimated values would be obtained. The most common method for TPC estimation is based on a modification of the Folin-Ciocalteu procedure. ${ }^{[16]}$ The TPC values based on the method that used Folin-Ciocalteu instead of the Folin-Denis reagent and gallic acid as the reference standard are less dependent on fluctuations and interference of non-phenols, and they are directly comparable to the "tannin" values obtained by the former 
Table 1. Total phenolic contents determined in this study and those reported by different groups in the literature for the same types of honey, all given as GAE (mg GA/kg honey)

\begin{tabular}{|c|c|c|c|c|}
\hline Reference & $\begin{array}{l}\text { Acacia } \\
\text { mean } \pm \mathrm{SD}(n)^{(\mathrm{a})} \\
\text { or } \min -\max (n)^{(\mathrm{a})}\end{array}$ & $\begin{array}{l}\text { Chestnut } \\
\text { mean } \pm \mathrm{SD}(n)^{(\mathrm{a})} \\
\text { or } \min -\max (n)^{(\mathrm{a})}\end{array}$ & $\begin{array}{l}\text { Lime } \\
\text { mean } \pm \operatorname{SD}(n)^{(\mathrm{a})} \\
\text { or } \min -\max (n)^{(\mathrm{a})}\end{array}$ & Country \\
\hline Our results $1^{(b)}$ & $186 \pm 36(19)$ & $561 \pm 114(12)$ & $382 \pm 108(8)$ & Cro/Ser/Hun/Ita \\
\hline Our results $2^{(c)}$ & $199 \pm 34(19)$ & $557 \pm 109(12)$ & $386 \pm 103(8)$ & Cro/Ser/Hun/Ita \\
\hline Our results $3^{\text {(d) }}$ & $171 \pm 36(19)$ & $554 \pm 117(12)$ & $371 \pm 110(8)$ & Cro/Ser/Hun/Ita \\
\hline Our results $4^{(\mathrm{e})}$ & $222 \pm 38(19)$ & $623 \pm 122(12)$ & $431 \pm 115(8)$ & Cro/Ser/Hun/Ita \\
\hline [17] & $55.2 \pm 2.8$ & $211.2 \pm 5.5$ & & Italy \\
\hline [22] & $113 \pm 5.6(3)$ & $194.6 \pm 11.7$ (3) & & Italy \\
\hline [21] & - & $129-213(15)$ & $66.2-121(10)$ & Croatia \\
\hline [23] & $0-142.2(29)$ & & $180.0-292.3(8)$ & Croatia \\
\hline$[24]$ & $216.1 \pm 6.3(3)$ & $430.9 \pm 26.8(3)$ & $408.8 \pm 10.5(3)$ & Croatia \\
\hline [6] & $44.8 \pm 14.8(10)$ & $199.9 \pm 31.1(10)$ & $83.7 \pm 14.3(10)$ & Slovenia \\
\hline [25] & $20-390(10)$ & & $160-380(4)$ & Romania \\
\hline [19] & - & - & $80.7 \pm 145.1(4)$ & Czech \\
\hline [8] & $160.2 \pm 27$ & $982.6 \pm 177.7(7)$ & $412 \pm 41(3)$ & Turkey \\
\hline [26] & $191.1(3)$ & $976.6(3)$ & $621(3)$ & Turkey \\
\hline
\end{tabular}

(a) Mean value and standard deviation (SD) of total phenolic content (TPC) values calculated for $n$ honey samples. In the second part of Table 1, in addition to mean $\pm S D$, in some cases min - max values are given. The number of honey samples measured is given in the parenthesis, when it was reported in a study/reference.

(b) TPC values determined by the use of the calibration line given in Eq. (1) where $y$ denotes the absorbance and $x$ is the concentration of gallic acid. (c),(d),(e) TPC values determined using Eqs. $(1-4)$, respectively.

standard method. ${ }^{[9]}$ Therefore, this method has become frequently used in analyses of TPC in biological samples and has been very often cited in the literature. ${ }^{[9,13,27]}$ TPC determination has been complemented by other methods capable of identifying individual polyphenolic compounds in samples that require calibration procedures based on chemicals as standards. ${ }^{[28,29]}$ The aforementioned method has been regularly used in analyses of TPC in honey samples, especially after works of Meda et al..[16] and Beretta et al., ${ }^{[17]}$ for TPC changes, ${ }^{[30]}$ but also in analyses of other samples of plant origin or food products (fruit nectars and juices) with more detailed description of calibration process. $^{[31]}$

In several studies, interference analysis of compounds other than polyphenols on TPC measurements was performed. [32] Thus, a low presence of chlorophyll (i.e. very weak peaks at $690 \mathrm{~nm}$ ) was detected in several honey samples. ${ }^{[33]}$ However, chlorophyll and other non-phenolic compounds that could interfere with the determination of absorbance in the measurement of TPC in honey samples using the FC method are present to a lesser extent. ${ }^{[34]}$ In addition, their contents (such as the percentage of sugar in honey) are approximately uniform and possibly proportional to TPC values in all types of honey, and the determination of the TPC of all honey samples could be influenced roughly equally. The variation of this undetermined contribution is too small to explain the large variations in honey samples published intensively over the last 15 years, especially the recent ones (Table 2). Thus, the absorbance measured by the FC method at 750-765 nm corresponds essentially to the total antioxidant potential of the analysed sample. ${ }^{[32,34]}$ This is also confirmed by the high values of the correlation coefficients between the TPC values of 31 plant samples and the antioxidant activities measured by four antioxidant capacity methods which showed the values of $0.94,0.97,0.83,0.91$ and 0.85 for DPPH, ABTS, ORAC, FRAP and SOD tests, respectively. ${ }^{[34]}$ Even higher and more significant correlations between TPC and FRAP $(r=0.97)$ and the DPPH method $(r=0.93)$ were found in the analysis of 70 honey samples from Slovenia. It was concluded that these correlation coefficients indicate that the antioxidant activities of these honey samples mainly come from their phenolic content. ${ }^{[6]}$ Similar results were obtained ( $r$ values between $0.87-0.93$ ) in a highly cited study by Beretta et al. ${ }^{[17]}$ (obtained $r$ values between $0.87-0.93$ ) and an analogous conclusion was reached in the analysis of 14 honey samples of different geographical origin. Therefore, the FC method is considered the most acceptable in determining TPC of samples of plant origin and honey samples. ${ }^{[34,35]}$

For the same uni-floral types of honey, the differences may be related to the different times of collection and spinning of honey by beekeepers, the different conditions and duration of storage, the geographical origin, as well as to 
the various equipment used and methodology/procedure applied during beekeeping. However, the differences may also be due to inconsistencies in the application of standardized measurement methods. ${ }^{[17]}$ Also, some authors stated that they modified the TPC measurement method, but without specifying exactly which part of the protocol has been changed. ${ }^{[17]}$ Also, in constructing the gallic acid calibration line, some authors used the interval 0 $-200 \mathrm{mg} / \mathrm{L}$ (Figure 1) ${ }^{[6,16]}$ while others used $10-250 \mathrm{mg} / \mathrm{L}$

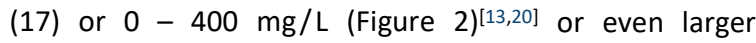
intervals. ${ }^{[18,19]}$ Very large variations of TPC of uni-floral honey samples in the literature are evident from Table 1. Because of that, and aiming to contribute to the standardization of TPC measurement, we analysed in this study the influence of the calibration concentration range and the procedure used to establish the calibration line on the TPC of uni-floral honey samples.

The GAE TPC in honey samples were determined using calibration lines in the GA concentration ranges $0.02-0.2 \mathrm{~g} / \mathrm{L}$ (Eqs. (1) and (2), Figure 1) and $0.04-0.4 \mathrm{~g} / \mathrm{L}$ (Eqs. (3) and (4), Figure (2)). In both concentration ranges, one calibration line is determined in free form containing the intercept (Eqs. (1) and (3)) and forcing the line to pass through the origin (Eqs. (2) and (4)). The slopes of these lines are very similar, but are closer in equations (1) and (2) which were determined in a narrower concentration range. The latter approach (i.e. the calibration line through the origin) is recommended in a very frequently cited paper by Molyneux[36] that provides an overview and recommendations for calibration procedures in the study of biological activity of chemical compounds being antioxidants. Namely, he recommended that the origin $(0,0)$ should be taken as multiple experimental points. Following the standard practice, the instrument is zeroed with solvent in a matching cuvette for each sample reading. Furthermore, Molyneux ${ }^{[36]}$ stated that when calibrating the spectrometer with solution only (when the sample concentration is 0 ), the absorbance value will be 0 . This procedure should be repeated once for each sample absorbance reading. The final suggestion was to force the linear regression line pass through the origin (with the constant being equal to 0). ${ }^{[36]}$ Thus, the calibration line will have the form equivalent to the standard form of BeerLambert relationship (Eq. (5)):

$$
\text { Absorbance }=\varepsilon \subset L
$$

where $\varepsilon$ is the extinction coefficient (properties of the solvent and sample), $c$ is the solute concentration ( $\mathrm{mol} / \mathrm{L}$ ), and $L$ is the path length (conventionally, $1 \mathrm{~cm}$ ). ${ }^{[36]}$ However, this is not accepted as the only correct approach. In the literature related to the measurements of TPC honey samples it has not usually been specified that the calibration lines are constructed in such a way.
The correlation coefficients for all these equations are very similar, with the differences in the fourth decimals. Having in mind that this is the main quality parameter given for calibration line in the literature, we see that this is not a suitable/useful criterion since it cannot be used to select the best line among several possible ones. The standard error of estimate $(S)$ given for each calibration line is shown to be a much more sensitive parameter. Moreover, the standard errors of the calibration line passing through the origin (Eqs. (2) and (4)) are $~ 93 \%$ and $56 \%$ (respectively) larger than for the corresponding lines that do not pass through the origin (Eqs. (1) and (3)). From these results, one can see that the calibration lines passing through the origin are not statistically optimal, and cause larger errors and constant or linear shifts. Such a shift is considered in the calculation of $S$, but not in the formula for $r$. This is consistent with the conclusion from the literature, ${ }^{[37]}$ where it was illustrated that the correlation coefficient is insensitive to a constant (or linear) shift between sets of correlated values (variables). These results show the need for the calculation and reporting in the literature the standard error related to each calibration line - as a more sensitive criterion of the fitting quality. Based on this analysis, we can conclude that a more reliable calibration line is the one described by a complete equation (Eq. (1) or Eq. (3)) containing a constant term (i.e. the intercept on the $y$-axis) because the standard error of estimate in fitting is lower in such a case.

By determining the concentration using these calibration lines, different TPC values are obtained. Comparing mean TPC values (determined separately for each honey floral-type) calculated using the calibration line without the constant term ( $y$-axis intercept) (Eqs. (2) and (4)) with the corresponding line containing the constant term (Eqs. (1) and (3)), it is clear that the former give higher TPC values (Table S2). The only exception is the calibration line for chestnut in the concentration range of $0.02-0.2$ $\mathrm{mg} / \mathrm{L}$. The differences in TPCs are much (several times) larger when a wider concentration range of $0.04-0.4$ $\mathrm{mg} / \mathrm{L}$ is used for the construction of calibration lines, i.e. between TPC values read from the Eqs. (3) and (4). On the other hand, the differences between maximal and minimal values determined by calibration Eq. (1) are higher than those determined by Eq. (2), and it is valid for TPC values for each of the three honey floral-types considered. However, mentioned finding is the opposite for wider concentration intervals (Eq. (3) and Eq. (4)), i.e. wider intervals of TPC values are obtained by using Eq. (4) for each honey floral type.

Taking a larger amount of honey in measurements, higher absorbance is also obtained. It is evident from Figure 1 , and especially from Figure 2, that the calibration line becomes nonlinear at minimal and maximal concentrations, 
Table 2. Total phenolic contents reported recently by different groups in the literature given in original units, and expressed as $\mathrm{GAE}$ (mg GA/kg honey)

\begin{tabular}{|c|c|c|c|c|}
\hline Ref. & $\begin{array}{l}\text { TPC, mg GA/kg } \\
\min -\max (n)^{(a)}\end{array}$ & in original units ${ }^{(b)}$ & honey type ${ }^{(c)}$ & Country \\
\hline This study & $171-623(39)$ & - & aca/lim/che & Cro/Ser/Hun/Ita \\
\hline (38) & $200-1000(7)$ & - & different & Italy \\
\hline (38) & $200(1)$ & - & acacia & Italy \\
\hline (39) & $755.2-2452.2(8)$ & $75.52-245.22 \mathrm{mg} \mathrm{GA} / 100 \mathrm{~g}$ & different & Morocco \\
\hline$(40)$ & $44-660(12)$ & $4.4-66 \mathrm{mg} \mathrm{GA} / 100 \mathrm{~g}$ & different & Serbia \\
\hline$(40)$ & $160-880(7)$ & $16-88$ mg GA/100g & different & different \\
\hline$(41)$ & $16.3-419.3(64)$ & - & different & India \\
\hline$(41)$ & $98.2-432.91(20)$ & - & different & Yemen \\
\hline$(42)$ & $0.01215-0.02645(3)$ & $12.15-26.45$ (3) $\mu \mathrm{g} \mathrm{GA} / \mathrm{kg}$ & different & Malaysia \\
\hline (43) & $1.3-7.39(60)$ & $0.13-0.739 \mathrm{mg} \mathrm{GA} / 100 \mathrm{~g}$ & different & Brazil \\
\hline
\end{tabular}

(a) min - max values are given for total phenolic content (TPC). The total number of honey samples measured is given in the parenthesis.

(b) The values and in units as it was reported originally.

(c) aca = acacia, lim = lime, che = chestnut

resulting in GAE concentration reading error. Such an error can lead to errors in TPC readings caused by the use of calibration lines like those given by Eqs. $(1-4))$. Therefore, it is recommended (if possible) that the sample absorbance is not at the ends of the range of gallic acid absorbances used in determining the calibration line.

\section{Comparison with TPC Values from the Literature}

From the analysis of the measured TPC values in Table 1, one can notice significant differences for the same type of honey, and that the differences do not depend on the country of honey origin. The mean TPC value for acacia determined by four calibration lines on 19 samples in this study is $194.5 \mathrm{mg} \mathrm{GAE} / \mathrm{kg}$, while other results from the literature listed in Table 1 (corresponding to honey samples from regions close to Croatia, Serbia, Hungary and Italy) are in the range $55.2-390 \mathrm{mg} \mathrm{GAE} / \mathrm{kg}$. For chestnut and lime, mean TPC values of 573.8 and $392.5 \mathrm{mg} \mathrm{GAE} / \mathrm{kg}$ (respectively) are determined, and the corresponding literature results from Table 1 are between 129 and 982.6 $\mathrm{mg} \mathrm{GAE} / \mathrm{kg}$ (chestnut) and 66.2 to $621 \mathrm{mg} \mathrm{GAE} / \mathrm{kg}$ (for lime). We obtained that the mean TPC values are the highest for chestnut honeys. The average TPC value for chestnut is approximately 1.5 and 3 times higher than that for lime and acacia, respectively. From these TPC values and their ranges, it is evident that our results are approximately in the middle of the literature ranges for each of the three analysed honey floral types. Evidently, the values from the literature differ greatly, and the ratio of the highest and the lowest TPC values for honey samples from the literature listed in Table 1 is usually about 10. As mentioned above, one of the reasons could be the calibration process, but also other reasons such as the storage conditions of honey, botanical or geographical origin, etc. ${ }^{[30]}$ Namely, in analyses of TPC changes during storage of honey very high variations were observed (for a factor of approx. 10) during storage of one year - according to the study by Šarić et al. ${ }^{[30]}$ (as presented in Table 2 on page 12).

Additionally, we compare our results for acacia, lime and chestnut honey with some other studies, regardless of their floral-type or regional origin (Table 2).

TPC values obtained in this study by considering four calibration lines and three honey types agree well with TPCs from most of the studies ${ }^{[38-41]}$. However, the range of TPC values from this study differs drastically from those given in two recent studies (from 2021) that provide results for honey samples from Malaysia and Brazil. In these cases, this may be an error in the calculation of units of measurement in which the TPC values are reported. This situation is more confusing because these two studies from papers ${ }^{[42,43]}$ were published in the same year (2021) and the same reputable journal as the two previous studies ${ }^{[40,41]}$ whose values are (mostly) in agreement with the results obtained in this paper, as well as with those from other studies ${ }^{[38,39]}$ whose TPC values are given in Table 2. Finally, this case/situation also points to the need to (1) refine standard procedures for determining TPC in samples of plant origin, including calibration line construction procedures, and (2) specify the description of procedures in the literature. 


\section{CONCLUSIONS}

The Follin-Ciocalteu method is based on the oxidation of phenolic group with phosphomolybdic and phosphotungstic acid and it is relatively sensitive. Also, TPCs in herbal and honey samples of the same origin can vary greatly for many reasons, as illustrated in this study on several unifloral honey samples from the literature (Table 1).

To make possible more strict comparisons of TPCS between different studies, the determination of TPC by the Folin-Ciocalteau method should be described with more precise details, so that the experiments can be reproduced by others. It is also necessary to specify precisely the location of honey, the period and conditions of storage, the time of honey production, improve the accuracy of floral origin detection, etc. All these and other factors, such as the form of the calibration line and the range in which it is determined, can influence the estimated TPC value of honey. By preparing gallic acid calibration lines in the ranges $0-0.2 \mathrm{mg} / \mathrm{L}$ or $0-0.4 \mathrm{mg} / \mathrm{L}$, it was shown that higher or lower TPC values are obtained. Furthermore, an additional variation of TPC was detected depending on whether the calibration line passes through the origin $(0,0)$ or not. Although the differences between the calibration lines in Figures (1) and (2) appear to be very small, we have shown that the differences in the TPC values of 39 honey samples are noticeable. However, consistent TPC values of acacia, chestnut and lime honey samples were obtained, showing that chestnut honey contains (on average) 3 times more polyphenolics than acacia, and 1.5 times more than lime, which is consistent with the vast majority of results from the literature.

The number of gallic acid samples and the range of concentrations used to construct of the calibration line should be adjusted in such a way that all absorbances are between the minimal and maximal values of gallic acid used for the determination of the calibration line. Moreover, it is recommended that the absorbances of honey samples should be closer to the middle of the calibration range and, if possible, be away from the end values. Formulae used for estimating TPC values from the calibration line should be given together with gallic acid concentrations and measured absorbances, statistical parameters of calibration line determined and other details needed for the computing units of TPC (mg GA/ kg, mg GA/g or mg GA/100 g).

The standard error of estimate in fitting ascribed to a calibration line is a more sensitive indicator of its quality than the correlation coefficient $(r)$. It is recommended that $S$ should be given for the calibration line used for the estimation of TPC. Moreover, it is easy to generalize and conclude that the same is valid for all other similar estimates and analyses that require a calibration line. To avoid other possible mistakes and to enable reproduction of TPC measurements, it is suggested to give (e.g. in supplements of published papers) in research studies all measured absorbances and concentrations used in computing the TPC and in determining the calibration line. By that data given in research studies, other researchers could re-calculate the calibration line in the way they consider the most appropriate and use it for re-estimation of honey TPC values to perform comparative analysis.

Acknowledgements. The authors were supported by the Croatian Ministry of Science and Education (D. Bešlo, D. Agić, B. Lučić and D. Vikić-Topić) through basic grants given to their institutions and by the Croatian Government, the European Union through the European Regional Development Fund - the Competitiveness and Cohesion Operational Programme (KK.01.1.1.01) The Scientific Centre of Excellence for Marine Bioprospecting - BioProCro (B. Lučić, through research activities in molecular modelling and model validation and theoretical work in the project module Bioinformatics).

Supplementary Information. Supporting information to the paper is attached to the electronic version of the article at: https://doi.org/10.5562/cca3805. Two tables are given as Supplementary information. Table S1 contains concentrations and absorbances (in triplicate) of gallic acid used for the determination of calibration lines. Table S2 contains details on honey samples (producers, floral and regional origin), honey sample absorbance values determined in the triplicate and TPC values calculated by Eqs. (1-4). Graphical illustrations of scattering of TPC values (mg GAE / kg honey) for acacia, chestnut and lime honey samples are given in Figures S1, S2 and S3, respectively.

PDF files with attached documents are best viewed with Adobe Acrobat Reader which is free and can be downloaded from Adobe's web site.

\section{REFERENCES}

[1] L. H. Cazarolli, L. Zanatta, E. H. Alberton, M. S. R. Bonorino Figueiredo, P. Folador, R. G. Damazio, M. G. Pizzolatti, F. R. M. Barreto Silva, Mini Rev. Med. Chem. 2008, 8, 1429-1440. https://doi.org/10.2174/138955708786369564

[2] R. J. Williams, J. P. E. Spencer, C. Rice -Evans, Free Radic. Biol. Med. 2004, 36, 838-849.

https://doi.org/10.1016/j.freeradbiomed.2004.01.001

[3] N Gheldof, X. H. Wang, N. J. Engeseth, J. Agric. Food Chem. 2002, 50, 5870-5377.

https://doi.org/10.1021/jf0256135

[4] A. Guler, A.Bakon, C. Nisbet, O. Yavuz, Food Chem. 2007, 105, 1119-1125.

https://doi.org/10.1016/j.foodchem.2007.02.024

[5] S. Saxena, S. Gautam, A. Sharma, Food Chem. 2010, 118, 391-397.

https://doi.org/10.1016/j.foodchem.2009.05.001

[6] J. Bertoncelj, U. Doberšek, M. Jamnik, T. Golob, Food Chem. 2007, 105, 822-828

https://doi.org/10.1016/j.foodchem.2007.01.060 
[7] M. Ciulu, N. Spano, M. I. Pilo and G. Sanna, Molecules. 2016, 21, 1-32.

https://doi.org/10.3390/molecules21040451

[8] Z. Can, O. Yildiz, H. Sahin, E. A. Turumtay, S. Silici, S. Kolayli, Food Chem. 2015, 180, 133-141. https://doi.org/10.1016/j.foodchem.2015.02.024

[9] V. L. Singleton, J. Rossi, Am. J. Enol. Vitic. 1965, 16, 144-158.

[10] S. Ouchemoukh, H. Louaileche, H. Schweitzer, H. Food Control. 2007, 18, 52-58. https://doi.org/10.1016/j.foodcont.2005.08.007

[11] A. M. Aljadi, M. Y. Kamaruddin, Food Chem. 2004, 85, 513-518. https://doi.org/10.1016/S0308-8146(02)00596-4

[12] F. Shahidi, V. Zhang, J. Funct. Foods. 2015, 18, 757781. https://doi.org/10.1016/j.jff.2015.01.047

[13] V. L. Singleton, R. Orthofer, R. Lamuela-Raventos, Methods. Enzymol. 1999, 299, 152-178. https://doi.org/10.1016/S0076-6879(99)99017-1

[14] M. Al-Mamary, A. Al-Meeri, M. Al-Habori, Nutr. Res. 2002, 22, 1041-1047. https://doi.org/10.1016/S0271-5317(02)00406-2

[15] U. Gašić, S. Kečkeš, D. Dabić, J. Trifković, D. Milojković-Opsenica, M. Natić, Ž. Tešić, Food Chem. 2014, 145, 599-607. https://doi.org/10.1016/j.foodchem.2013.08.088

[16] A. Meda, C. E. Lamien, M. Romito, J. Millogo, O. G. Nacoulma, Food Chem. 2005, 91, 571-577. https://doi.org/10.1016/j.foodchem.2004.10.006

[17] G. Beretta, P. Granata, M. Ferrero, M. Orioli, R. M. Facino, Anal. Chim. Acta. 2005, 533, 185-191. https://doi.org/10.1016/j.aca.2004.11.010

[18] J. Piljac, S. Martinez, L. Valek, T. Stipčević, K. Kovačević Ganić, Food Technol. Biotechnol. 2005, 43, 271-276.

[19] B. J. Xu, S. K. C. Chang, J. Food Science. 2007, 72, S159-S166.

https://doi.org/10.1111/j.1750-3841.2006.00260.x

[20] J. Lachman, A. Hejtmankova, J. Sykova, J. Karban, M. Orsak, B. Rygerova, Czech. J. Food Sci. 2010, 28, 412426.

https://doi.org/10.17221/202/2009-CJFS

[21] I. Flanjak, D. Kenjerić, D. Bubalo, Lj. Primirac, Eur. Food Res. Techol. 2016, 242, 467-475. https://doi.org/10.1007/s00217-015-2557-0

[22] E. Pichichero, L. Canuti, A. Canini, J. Sci. Food Agric. 2009, 89, 609-616. https://doi.org/10.1002/jsfa.3484

[23] G. Šarić, K. Marković, N. Major, M. Krpan, N. UršulinTrstenjak, M. Hruškar, N. Vahčić, Czech. J. Food Sci. 2013, 31, 601-606. http://doi.org/10.17221/509/2012-CJFS

[24] J. Piljac-Žegarec, T. Stipčević, A. Belščak, J. ApiProduct ApiMedical Sci. 2009, 1, 43-50 https://doi.org/10.3896/IBRA.4.01.2.04
[25] M. L. Al, D. Daniel, A. Moise, O. Bobis, L. Laslo, S. Bogdanov, Food Chem. 2009, 112, 863-7. https://doi.org/10.1016/j.foodchem.2008.06.055

[26] Ş Kivirak, I. Kivirak, E. Karababa, Food Sci. Technol. Campinos. 2017, 37, 80-89. https://doi.org/10.1590/1678-457x.07916

[27] G. Marigo, Analysis, 1973, 2, 106-110.

[28] M. Medvidović-Kosanović, A. Šter, L. Jakobek, P. Krivak, R. Mađarić, Croat Chem. Acta. 2017, 90, 129134. https://doi.org/10.5562/cca3120

[29] M. Kondža, H. Rimac, Ž. Maleš, P. Turčić, I. Ćavar, M. Bojić, Croat. Chem. Acta 2020, 93, 33-39. https://doi.org/10.5562/cca3652

[30] G. Šarić, K. Marković, N. Major, M. Krpan, N. UršulinTrstenjak, M. Hruškar, N. Vahčić, Food Technol. Biotechnol. 2012, 50, 434-441.

[31] S. Inić, M. Ljepović, A. Domijan, S. S. Srdarević, J. Jablan, K. Markov, Croat. Chem. Acta, 2020, 93, 57-62. https://doi.org/10.5562/cca3662

[32] J. D. Everette, Q. M. Bryant, A. M. Green, Y. A. Abbey, G. W. Wangila, R. B. Walker, J. Agric. Food Chem. 2010, 58, 8139-8144. https://doi.org/10.1021/jf1005935

[33] E. Parri, G. Santinami, V. Domenici, Appl. Sci. 2020, 10, 1776. https://doi.org/10.3390/app10051776

[34] S. Dudonné, X. Vitrac, P. Coutière, M. Woillez, J.-M. Mérillon, J. Agric. Food Chem. 2009, 57, 1768-1784. https://doi.org/10.1021/10.1021/jf803011r

[35] S. Kupina, C. Fields, M. C. Roman, S. L. Brunelle, Journal of AOAC INTERNATIONAL 2018, 101, 1466. https://doi.org//10.5740/jaoacint.18-0031

[36] P. Molyneux, Songklanakarin J. Sci. Technol. 2004, 26, 211-219.

[37] B. Lučić, J. Batista, V. Bojović, M. Lovrić, A. Sović Kržić, D. Bešlo, D. Nadramija, D. Vikić-Topić, Croat Chem. Acta. 2019, 92, 379-391. https://doi.org/10.5562/cca3551

[38] A. Rosa, C. I. G. Tuberoso, A. Atzeri, M. P. Melis, E. Bifulco, M. A. Dessì, Food Chem. 2011, 129, 1045-1053. https://doi.org/10.1016/j.foodchem.2011.05.072

[39] R. El-Haskoury, W. Kriaa, B. Lyoussi, M. Makni, J. Food Drug Anal. 2018, 26, 67-73. https://doi.org/10.1016/j.jfda.2016.11.016

[40] D. Velimirović, S. Tošić, S. Mitić, A. Pavlović, I. Rašić Mišić, G. Stojanović, J. Apicult. Res. 2021, 1-13. https://doi.org/10.1080/00218839.2021.1898783

[41] M. A. Saeed, M. Jayashankar, H. K. Tejaswini, J. Apicult. Res. 2021, 1-14. https://doi.org/10.1080/00218839.2021.1898835

[42] N. I. I. Muhammad, N. M. Sarbon, J. Apicult. Res. 2021, 1-8. https://doi.org/10.1080/00218839.2021.1896214

[43] M. D. Archilia, A. A. L. Neto, M. C. Marcucci, R. C. B. Alonso, T. C. de Camargo, R. C. Camargo, A. C. H. Frankland Sawaya, J. Apicult. Res. 2021, 1-12. https://doi.org/10.1080/00218839.2021.1886747 